\title{
Marsupialization of a large pediatric mandibular cyst with a multipurpose space maintainer, a prospective study
}

\section{Original Article}

\author{
Salem Waly \\ ${ }^{a}$ Department of Oral and Maxillofacial Surgery, Faculty of Dental Medicine, \\ Al-Azhar University, Cairo, Egypt
}

\begin{abstract}
Background: Radical cysts are the most common inflammatory odontogenic cystic lesions that can be treated by Marsupialization, especially if the cyst is large and has vital structures. There are a few things to consider when treating a growing patient with an odontogenic cyst. Marsupialization has more benefits for the growing patient than enucleation to overcome treatment morbidity, such as damage to vital structures, bone loss, jaw growth disorders and pathological fractures.

Purpose: This article reports on the success of marsupialization of odontogenic cysts "that have been associated with very decaying deciduous molars" to reduce treatment morbidity and to discuss enucleation in young patients.

Materials and Methods: A healthy 9 patients with average age of 8 years old with unilateral radicular mandibular cysts. Marsupialization was done before the end of cyst enucleation incorporating a multipurpose space maintainer.

Results: Periodic contraction of the cysts was observed in all patient cystic site. The position of the cyst wall was placed superficially and an enucleation was performed. The treatment result was satisfactory with low morbidity.

Conclusion: Marsupialization with a versatile space maintainer for the odontogenic periapical cyst in growing patients has more advantages than enucleation in reducing treatment morbidity.
\end{abstract}

Key Words: Marsupialization, Periapical cyst and Space maintainer.

Received: 07 April 2020, Accepted: 13 April 2020.

Corresponding Author: Salem Waly, Department of Oral and Maxillofacial Surgery, Faculty of Dental Medicine, Al-Azhar University, Cairo, Egypt, Tel.: +966560943242, Mobile:+966560943242, E-mail: salemwaly2006@yahoo.com.

ISSN: 2090-097X, October 2019, Vol. 10, No. 4

\section{INTRODUCTION}

Odontogenic cysts are common in daily practice. They are generally divided into two types, the developmental cyst and the inflammatory cyst. The odontogenic cyst comes from the rest of the Malassez epithelium, which multiplies to form the cyst wall. An odontogenic cyst can be enlarged by destroying the extracellular matrix with collagenase, bone resorption around the cyst from osteoclast-like cells, or a combination of both ${ }^{[1]}$.

In addition to the enlarged cyst, the intracystic pressure also increases, which helps with the enlargement. In addition to mechanical pressure, cytokines such as interleukin-1 $\alpha$ (IL-1 $\alpha)$ and interleukin-6 (IL-6) play an important role in enlarging the cyst ${ }^{[2]}$.

The periapical cyst is the most common inflammatory odontogenic cyst. It is more common in the permanent dentition, but rarely in the primary dentition ${ }^{[3]}$. The periapical cyst is caused by irritation of the periapical tissue due to pulp necrosis due to cavities or trauma that could stimulate the proliferation of the Malassez epithelium. The incidence of the periapical cyst is most common in the second decade of life. Permanent teeth have an incidence of $52 \%$ to $68 \%$ and are less common in primary teeth that make up 0.5 to $3.3 \%$ of all cysts ${ }^{[4]}$.

There are certain techniques for treating the odontogenic cyst, i.e. enucleation, marsupialization or a combination of these techniques. Enucleation is the complete removal of the cystic wall. A small cyst is usually enucleated in conjunction with an apicoectomy or tooth extraction. Marsupialization is indicated for a large mandibular cyst that is close to an important structure such as the mandibular canal or the mental neurovascular bundle ${ }^{[5]}$. Consequently, the osmotic cystic pressure would disappear after marsupialization; As a result, the bone around the lesion could regenerate and the size of the cyst would be reduced.

The treatment of the periapical cyst for the growing patient must take certain things into account, such as permanent tooth buds, patient cooperation, jaw growth and the healing process. The enucleation of a large cyst has high morbidity and is dangerous for the most important bone structures, blood vessels and nerve bundles. The small or medium-sized periapical cyst of the jaw, especially those that are still covered with thick cortical bone, would cause difficulties in reaching the cyst without serious bone damage. Access from the extraction site is usually poor and it is difficult to ensure that the entire cyst has been removed. Marsupialization is said to stimulate the regeneration potential of bones and soft tissues, especially in young patients ${ }^{[6 \text { and } 7]}$.

The above reasons have shown that marsupialization is the treatment of choice to avoid bone defects, neurovascular 
damage, which is beneficial for young patients with thick cortical bones. Given the interest of critical stage of mixed dentition in children, which varies between 5.5 and 12 years, the sequential shedding of the quadrant of the deciduous teeth is one of the important factors that lead to an adequate eruption of the permanent successors ${ }^{[8-10]}$.

If early deciduous teeth are lost, the entire mesiodistal width of the deciduous teeth must be maintained in order to avoid inadequate arch length. Therefore, space maintainers are used to temporarily occupy this space to avoid wasting space $^{[11]}$. In contrast, the retained deciduous teeth may interfere the normal permanent teeth eruption path into the dental arch. A similar effect can also be caused by cysts, tumors that cause a malocclusion and permanent jaw damage until controlled by the professional surgeons ${ }^{[12]}$.

Large cysts can be a problem in the mixed dentition stage, since cystic pressure can lead to deviations in the developing tooth buds and in some cases, permanent teeth eruption could be impaired ${ }^{[13}$ and ${ }^{14]}$. This article reports a case series study of mandibular periapical cyst with thick cortical bone. For those cases, a marsupialization treatment with versatile space maintainer was selected before enucleation as a promising efficient management modality.

This study was aimed to evaluate the effect of osseodensification technique on primary stability in comparison to the conventional drilling technique.

\section{MATERIALS AND METHODS}

It is a prospective case series study from June 2017 to May 2019. This clinical study included 9 consecutive pediatric patients have had visited the outpatient department at Al Ansari Special Hospital in Saudi Arabia. Out of the group of patients, there were 8 female and 1was male with average age 8 years old. All patients in the study with a standard severe complaint about persistent pain and swelling in the molar region of the lower jaw.

Inclusion criteria were as all pediatric patients with mandibular cystic lesion. Exclusion criteria: The patients who were having medical compromises of immunodeficiency, autoimmune disease, radio or chemotherapy or trauma were excluded, as they were not the ideal candidate for ideal wound healing capacity.

The intraoral examination of 2 cases revealed badly decayed primary molars with previous non-convenient root canal treatment. The remaining 7 cases were badly broken nonrestorable primary molar teeth. A detailed history has shown that all of these deciduous molars have been extremely decaying and painful for more than 3 to 6 month before seeking the treatment.

Panoramic radiography and computed tomography had been taken for all cases, showed a huge radiolucency under the deciduous molars (Figures 1 and 2). Premolar tooth buds are involved in radiolucent area and the path of eruption has been redirected in all cases. Based on the medical history, clinical and radiological examination, a preliminary diagnosis of the radicular cyst was made and the medical history was continued without significance.

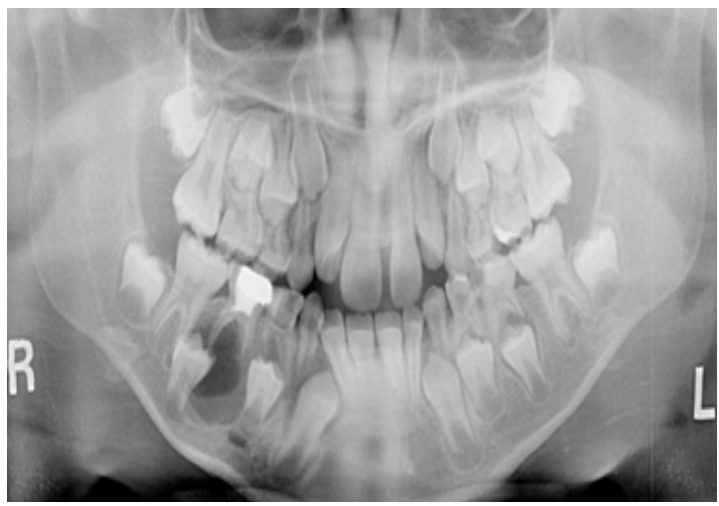

Figure 1: Panoramic radiography showed huge radiolucency under lower right deciduous molars.

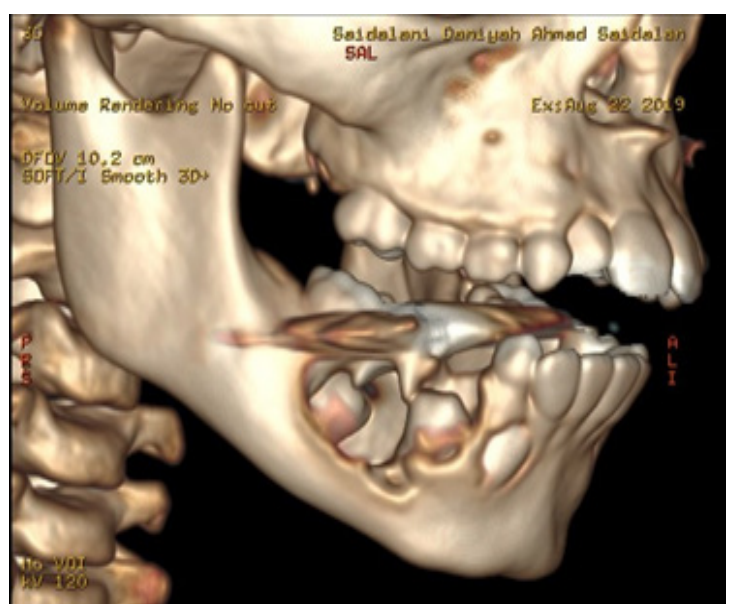

Figure 2: Computed tomography showed huge radiolucency under lower right deciduous molars. 
The following information was collected and tabulated from the individual patient's medical records including: age, gender, duration of cystic cavity, the existence of infection, and period between registered complain and total recovery was searched (Table 1).

Table 1: Characteristics of patients, treatments and results

\begin{tabular}{|c|c|c|c|c|c|c|}
\hline Patients & Age (years) & Gender & Treatment Period & $\begin{array}{l}\text { Contributing } \\
\text { Local Factor }\end{array}$ & Patient Compliance & Approach \\
\hline 1 & 8 & female & 3 month & badly broken tooth & under L A & \\
\hline 2 & 9 & female & 3 month & inconvenient RCT & under L A & \\
\hline 3 & 7 & female & 3 month & badly broken tooth & under L A & \\
\hline 4 & 8 & male & 3 month & badly broken tooth & under L A & marcipulization \\
\hline 5 & 7 & female & 6 month & inconvenient RCT & under G A & w MPSM \\
\hline 6 & 9 & female & 3 month & badly broken tooth & under L A & \\
\hline 7 & 8 & female & 4 month & badly broken tooth & under $\mathrm{G} \mathrm{A}$ & \\
\hline 8 & 7 & female & 3 month & badly broken tooth & under L A & \\
\hline 9 & 9 & female & 3 month & badly broken tooth & under L A & \\
\hline
\end{tabular}

Abbreviations: RCT (root canal treatment), MPSM (multipurpose space maintainer), LA (local anesthesia), GA (general anesthesia).

The postoperative complications, as well as the need of reoperation were registered. The obtained data from our charts were submitted to descriptive statistical analysis for the purpose of this article.

\section{The treatment protocol}

The offending deciduous molars were removed and the marsupialization was performed under local anesthesia in 6 cases while 3 non cooperative cases had been managed under general anesthesia, the roof surface of the lesion was enucleated and the intracystic pressure was reduced by removing the cystic fluid with a sterile syringe. The produced bone cavity was filled with an iodoform-based vaselinized gauze pad for two days. The edges of the cystic capsule were sutured to the oral mucosa.

The gauze was allowed to stand for 2 days after which an alginate impression was made and an acrylic obturator was fabricated. The design features included an acrylic plate with centric fenestration with a posterior stainless steel clasps on the right first permanent molar and anterior lingual plate (Figure 3).

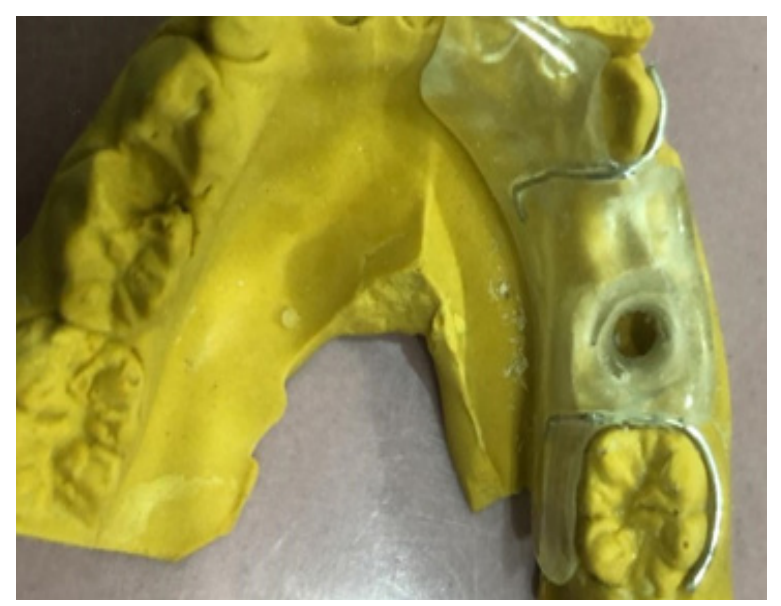

Figure 3: The design features multipurpose space maintainer.
On insertion of acrylic obturator splint, the stitches are removed. Care was taken to ensure that the splint covers the entire mesiodistal width of the 1st and 2nd lost primary molars and acts as a space maintainer. The postoperative period was without incident. The component was frequently checked every week and the splint was adjusted to make room for the 1st premolar tooth and the remaining part contained space for the second premolar. It had been remained in the mouth until the premolar eruption, which became visible through subsequent radiological evaluation (Figures 4).

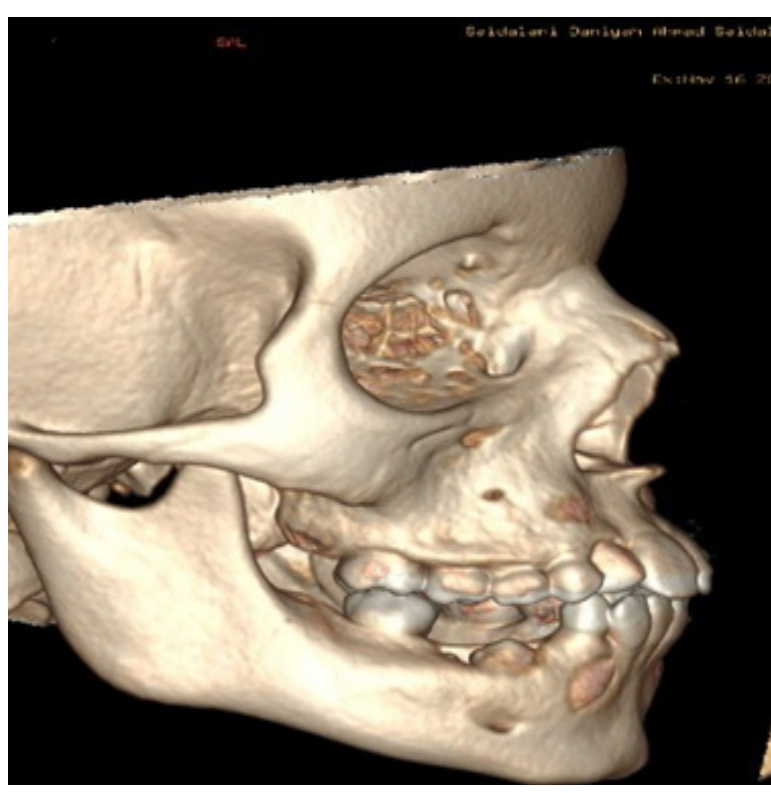

Figure 4: Complete mass resolution and 1st premolar eruption, which became visible through radiological evaluation. 


\section{RESULTS}

Nine pediatric patients with mandibular periapical cyst diagnosis were surgically treated at Al Ansari Special Hospital in Saudi Arabia from June 2017 to May 2019. The mean age of the study population was 8 years old (range, $6-9)$, and divided into 8 female cases $(88.9 \%)$ and 1 male (11.1\%) patient.

The periapical cysts were mainly caused by presence of non-restored badly broken primary teeth (7 patients, $77.8 \%$ ). The rest of the cysts were caused by inconvenient root canal treatment (patients, $22.2 \%$ ). All group of patients treated with marcipulization utilizing a multipurpose space maintainer and followed by cyst enucleation (Table 1).

The mean time registered between the persistence of cystic swelling and noneventful recovery was 3.4 month. Most of the cystic cases were approached in the dental office under local anesthesia $(7 ; 77.8 \%)$, while two uncooperative scared cases had been operated under general anesthesia $(2 ; 22.2 \%)$.

On late follow up in the period of 3 to 4 month, cyst reduction and marcipulization was considered as satisfactory in $88.9 \%$. There was one case $(11.1 \%)$ of wound infection that required an extended curing period up to 6 month. This complication of extended treatment period was considered to be due to insufficient self-oral hygiene measures. As a matter of fact, there was no case of the study group required a reoperation.

\section{DISCUSSION}

There are more options for the development and progression of caries in the milk dentition compared to the permanent dentition, which lead to pulpitis and periapical infection. The reasons for this are the decrease in the mineral content of the primary teeth compared to permanent teeth, eating habits and neglect due to the age factor ${ }^{[15]}$. If the carious lesion persists, it can cause a periapical abscess, which can lead to a periapical cyst. Bone mineralization is also relatively less in children than in adults. As a result, the cyst can quickly enlarge compared to adults ${ }^{[16-18]}$.

For a stable and ideal occlusion during the mixed dentition phase, it is important to maintain the correct eruption pattern and the development sequence of the permanent dentition. If there is an obstacle to the eruption, the developing tooth can change its eruption path or, in severe cases, get impacted. In this case, the periradicular cyst blocked the pathway of permanent premolar eruption, which required early diagnosis and treatment ${ }^{[18]}$.

Each surgical technique has several advantages and disadvantages for the treatment of the periapical cyst. The use of a particular surgical technique depends on the location, size, etiology, properties and their criteria ${ }^{[13]}$. At the mixed dentition stage, the treatment of choice for a small cyst is enucleation. However, if the cyst is closely related to large oral vital structures, marsupialization is the treatment method recommended ${ }^{[14,19]}$.

Marsupialization is preferred due to the lower morbidity and the fact that bone growth occurs as the lesion becomes smaller, resulting in a more normal bone contour, although long postoperative treatment is required, and the patient is strained. Reducing intracystic pressure is a key factor in the healing process ${ }^{[19]}$. In this case, marsupialization was preferred and performed because the cyst was closely related to the developing premolars tooth germ.

After surgery, iodoform was conditioned to reduce post-operative pain and infection. This gauze also retained the temporarily patented opening for the first two days until the multipurpose space maintainer was inserted ${ }^{[20]}$.

The fenestration must be kept open to keep the cystic pressure at a reduced level, which allows for bone regeneration and restoration, which leads to a reduction in the cystic size. Common techniques include suturing of the cavity cystic lining with the mucosal epithelium or making an obturator stent ${ }^{[21]}$.

In cases where the postoperative healing phase lasts longer, the custom obturator splint is preferred to the suturing technique. Therefore, in this case, a multipurpose obturator splint was made to maintain the opening patent and to keep the intracystic pressure at a reduced level, which favors non-erupted teeth to follow their normal eruption path ${ }^{[22}$ and 23].

A rapid loss of space occurs in the first 3 weeks after the extraction of the primary molars therefore, a maintenance of the space is necessary to avoid a reduction in the length of the arch due to the mesial drift of the first permanent $\operatorname{molar}^{[24]}$.

In this case, the multipurpose obturator splint maintained the entire mesiodistal width of the missing primary molars, thereby avoiding the mesial drift of the $1 \mathrm{st}$ permanent molar.

The multipurpose space maintainer had to be filled and trimmed regularly to reduce the size of the windows, which ultimately led to the closing of the lumen. This is caused by a decrease in intracystic pressure, continuous bone growth and metaplasia of the cystic lining, as well as the change from the lining of the cyst to be an oral epithelial lining. As a result, the obturator splint was filled regularly until the premolars appeared in the oral cavity ${ }^{[25}$ and 26$]$.

\section{CONCLUSION}

Despite the limited number of cases and short followup time in this clinical case series trial, it was concluded that Marsupialization followed by an acrylic obturator splint including of the space maintainer features is the preferred treatment, especially in cases where there is a large periapical cyst with primary molars. 


\section{CONFLICT OF INTEREST}

The authors declare no conflict of interest.

\section{REFERENCES}

1. Kubota $\mathrm{Y}$, Imajo I, Itonaga $\mathrm{R}$, Takenoshita $\mathrm{Y}$ (2013) Effects of the patient's age and the size of the primary lesion on the speed of shrinkage after marsupialisation of keratocystic odontogenic tumors, dentigerous cysts, and radicular cysts. $\mathrm{Br}$ J Oral Maxillofac Surg 51: 358 - 362.

2. Meghji S, Qureshi W, Henderson B, Harris M (1996) The role of endotoxin and cytokines in the pathogenesis of odontogenic cysts. Arch Oral Biol 41: 523 - 551 .

3. Sakurai A, Taniguchi Y, Hayashibara T, Senzui S, Homma H, Hashida T, Ooshima T, Shintani $\mathrm{S}$ (2011) Two cases of less invasive treatment for radicular cyst in primary dentition. Ped Dent J 21: $78-84$.

4. Kumar V, Sinha RK (2014) Radicular cyst in mixed dentition. J Stomat Occ Med 7: $85-89$.

5. Delilbasi C, Yuzbasioglu E, Aydin K (2012) Complete healing of radicular cysts only by marsupialization. J Clin Case Rep 2: 199.

6. Allon DM, Allon I, Anavi Y Kaplan I , Chaushu G (2014) Decompression as a treatment of odontogenic cystic lesion in children. JOMS 10: $24-31$.

7. Maksoud C, Piral T, Princ G, Guilhaume A (2012) Découverte fortuite d'un volumineux kyste mandibulaire chez un enfant. Arch Pediatr 9: $598-601$.

8. Harokopakis-Hajishengalis E (2007) Physiologic Root Resorption in Primary Teeth, Molecular Events and Histological Events: A Review. J Oral Sci 49:1 - 12 .

9. Sahara N, Okafuji N, Toyoki A, shizawa, Y. Yagasaki, H. Deguchi T, Suzuki K (1993) A histological study of the exfoliation of human teeth. J Dent Res 72: $634-640$.

10. Kumar CL, Sridhar MS (1990) Estimation of the age of an individual based on the times of eruption of permanent teeth. Forensic Sci Int 48:1 - 7 .

11. Bijoor RR, Kohli K (2005) Contemporary space maintenance for the pediatric patient. N Y State Dent J 71:32 - 37 .
12. Dibiase AT, Leggat TG (2000) Primary failure of eruption in the permanent dentition of siblings. Int J Paediatr Dent 10: 153 - 157.

13. Sokler K, Sandev S, Grgurevi J (2001) Surgical Treatment of Large Mandibular Cysts. Acta Stomatol Croat 35: 253 - 257.

14. Bodner L (2002) Cystic lesions of the jaws in children. Int J Pediatr Otorhinolaryngol 62: 25 - 9.

15. Li Y, Wang W (2002) Predicting caries in permanent teeth from caries in primary teeth: an eight-year cohort study. J Dent Res 81: $561-6$.

16. Boot AM, de Ridder MA, Pols HA, Krenning EP, Schrama SM (1997) Bone Mineral Density in Children and Adolescents: Relation to Puberty, Calcium Intake, and Physical Activity. J Clin Endocrinol Metab 82: 57 - 62.

17. Jovanovic G, Buric N, Mihailovic D, Tijanic M, Spasic M, Tarana S (2009) A radicular cyst in lower deciduous molar. Acta Stomatologica Naissi 23: $901-6$.

18. Gandhi S, Franklin DL (2008) Presentation of a radicular cyst associated with a primary molar. Eur Arch Paediatr Dent; 9: 56 - 9.

19. Riachi F, Tabarani C. (2010) Effective Management of Large Radicular Cysts Using Surgical Enucleation vs. Marsupialization Two Cases Report. Int Arab J Dent 1: 45 - 51.

20. Shear M, Speight P. (1992) Radicular and residual cysts. In: Cysts of the oral region, 3rd ed. Bristol: Wright 136-62.

21. Neaverth EJ, Burg HA (1982) Decompression of large periapical cystic lesions. J Endod 8:175-82.

22. Takase T, Wada M, Nagahama F, Yamazaki M (1996) Treatment of large radicular cysts by modified marsupialization. J Nichon Univ Sch Dent 98:161-8.

23. Sakkas N, Schoen R, Schulze D, Otten JE, Schmelzeisen R (2007) Obturator after marsupialization of a recurrence of a radicular cyst of the mandible. Oral Surg Oral Med Oral Pathol Oral Radiol Endod 103:16-8.

24. Park K, Jung DW, Kim JY (2009) Threedimensional space changes after premature loss of a maxillary primary first molar. Int J Paediatr Dent 19:3839-. 